\title{
Oral health-related quality of life in patients undergoing chronic hemodialysis
}

This article was published in the following Dove Press journal:

Patient Preference and Adherence

\author{
Ewa Rodakowska' \\ Magdalena Wilczyńska- \\ Borawska $^{2}$ \\ Justyna Fryc ${ }^{3}$ \\ Joanna Baginska ${ }^{4}$ \\ Beata Naumnik ${ }^{5}$ \\ 'Department of Restorative Dentistry, \\ Medical University of Bialystok, \\ Bialystok, Poland; ${ }^{2}$ Department of \\ Social and Preventive Dentistry, \\ Medical University of Bialystok, \\ Bialystok, Poland; ${ }^{3}$ Faculty of Medicine, \\ Medical University of Bialystok, \\ Bialystok, Poland; ${ }^{4}$ Department of \\ Dentistry Propaedeutics, Medical \\ University of Bialystok, Bialystok, \\ Poland; ' I Department of Nephrology \\ and Transplantation with Dialysis \\ Unit, Medical University of Bialystok, \\ Bialystok, Poland
}

Correspondence: Ewa Rodakowska Department of Restorative Dentistry, Medical University of Bialystok, ul Marii Skłodowskiej-Curie 24 A,

15-276 Bialystok, Poland

Tel +48 8574685760

Email ewarodakowska@interia.pl
Aims: The aims of the study were to determine oral health-related quality of life (OHRQoL) in chronic hemodialysis (HD) patients and to estimate which scale describing OHRQoL, Oral Health Impact Profile (OHIP-14) or Geriatric/General Oral Health Assessment Index (GOHAI), was more useful in this particular group.

Methods: This was a cross-sectional study conducted by means of a census survey. The Polish versions of OHIP-14 and GOHAI were used to assess OHRQoL. The oral examination included decayed, missing and filled teeth (DMF-T) Index; Oral Hygiene Index simplified; Plaque Index and Gingival Index. In the statistical analysis, the Kruskal-Wallis test, Mann-Whitney $U$ test, Pearson's $\chi^{2}$ test and Spearman's rank correlation coefficients were used as appropriate.

Results: The final sample consisted of 72 patients (mean age $63.2 \pm 15.2$ years). The mean duration of HD treatment was 43.8 months. The mean number of teeth was 10.9 . The majority of participants $(81.9 \%)$ were dentate; only $22.2 \%$ of the respondents had $>20$ teeth. Among the dentate subjects, $44.1 \%$ wore removable dental prostheses ( $60.7 \%$ women). The most prevalent items for GOHAI (mean 14.71; SD 7.21) were uncomfortable to swallow, discomfort when eating and unhappy with appearance. The most prevalent items for OHIP-14 (mean 8.87; SD 10.95) were uncomfortable to eat foods, and diet has been unsatisfactory. The internal reliability (Cronbach's alpha) was 0.637 for GOHAI and 0.918 for OHIP-14. Chewing problems were significantly related to GOHAI $(p=0.001)$ and OHIP-14 $(p<0.001)$ scales. Higher OHIP-14 scores were significantly associated with dental treatment needs $(p=0.029)$ and poor self-rated oral status $(p=0.001)$.

Conclusion: The HD patients had an unsatisfactory oral status, but using only OHRQoL scale was insufficient to capture all their oral health problems. The scales did not fully reflect poor oral health in HD patients. The oral problems were not a major concern for this group of patients, which could indicate the adaptation to impaired oral health or a change in health priorities. Regular dental examinations together with the assessment of OHRQoL in HD patients are required for a comprehensive patients' state. In our study, more variables were significantly related to the OHIP-14 scale than to the GOHAI scale. Thus, the OHIP-14 scale may be more useful in assessing OHRQoL in HD patients.

Keywords: oral health-related quality of life, OHIP-14, GOHAI, hemodialysis, OHRQoL

\section{Introduction}

The currently applicable definition of health according to the World Health Organization (WHO) emphasizes the significance of patients' well-being. ${ }^{1}$ It means that in health issues, we should concentrate not only on diseases but also on improving patients' well-being. At present, the need to assess the subjective well-being of patients, especially those with severe chronic diseases, is increasing. It was observed that oral status and systemic health are closely related. Impaired general health may 
affect oral health and vice versa. The maintenance hemodialysis (HD) patients are more susceptible to infections due to general debilitation, impaired immunologic response and chronic inflammation state. The inflammation, present also in the oral cavity, can take a more severe course due to the main illness and comorbid diseases.

A spectrum of oral manifestations in dialysis patients may be observed in the oral cavity. They include changes in periodontium (gingivitis, periodontitis), oral mucosa, salivary glands (xerostomia) and teeth. Periodontitis is considered to be a source of inflammation and a contributor to infectious diseases through the episodes of bacteremia and atherosclerotic complications. ${ }^{2-6}$ High prevalence of periodontitis is observed in patients with the kidney disease in both early and end stages. ${ }^{2,3,7-9}$ The aspect of complications present in the oral cavity requires detailed research because there are conflicting data presenting poor oral health and periodontitis ${ }^{4,10-13}$ as well as no increase in periodontitis in HD patients. ${ }^{14,15}$

Oral health-related quality of life (OHRQoL) is an integrated part of general health. Its outcomes may have important applications for clinical dentistry (orthodontic, periodontal therapy), dental research and health care. ${ }^{16,17}$ OHRQoL has a great impact on an individual's condition, and functional and emotional well-being, and is regarded as a part of the Global Oral Health Program. ${ }^{18}$ There are several measures assessing the OHRQoL. Among them, the most comprehensive and widely used are the Oral Health Impact Profile (OHIP-14) and the Geriatric/General Oral Health Assessment Index (GOHAI). ${ }^{19,20}$ The OHIP-14 includes functional limitation, physical pain, psychological discomfort, physical disability, psychological disability, social disability and handicap. ${ }^{19,21}$ The GOHAI measure evaluates three dimensions of OHRQoL including physical functions, psychosocial functions, pain and discomfort. ${ }^{20}$ The scales refer to different time periods: GOHAI to 3 months and OHIP-14 to 1 year. The studies on OHRQoL comprise mainly healthy patients at different ages. However, there are limited studies regarding the OHRQoL in patients with chronic diseases, especially those undergoing HD. What is more, we found hardly any publication comparing two scales describing OHRQoL in HD patients. ${ }^{9}$

The aims of the study were to determine OHRQoL in HD patients and to estimate which scale, OHIP-14 or GOHAI, was more useful in describing the OHRQoL in maintenance HD patients. In view of the fact that the GOHAI scale referred to 3 months and the OHIP-14 scale to 1 year, it was suggested that it would be better to use the scale describing
OHRQoL in the longer term. ${ }^{22}$ This may be crucial for the proper assessment of oral health, especially in patients with chronic diseases. To the best of our knowledge, this study is one of the first conducted in Europe that estimates which scale describing OHRQoL is more useful in maintenance HD patients.

\section{Methods}

\section{Study population}

The cross-sectional study was conducted in conformity with the Declaration of Helsinki and was approved by the ethical committee of the Medical University of Bialystok (R-I-002/317/14), Poland. The participation in the study was both anonymous and voluntary, and started after a written informed consent of the participants. The data were gathered by means of a census survey. All 92 patients who were undergoing maintenance HD at the I Department of Nephrology and Transplantation with Dialysis Unit of the University of Bialystok were included in the study. The final sample consisted of 72 participants. The response rate was $78.2 \%$. The inclusion criteria comprised edentulous patients and patients with few teeth because the missing teeth negatively influenced the OHRQoL. ${ }^{23}$ The exclusion criteria comprised patients who did not provide a written consent $(n=3)$, were unable to fill the whole questionnaire $(n=12)$ and were unable to comprehend the questionnaire $(n=5)$. The examination protocol was performed during two subsequent sessions. The demographic data were obtained, and the GOHAI questionnaire was filled by each participant during the first session. The oral examination was carried out by an experienced dentist at the bedside, and the OHIP-14 questionnaire was filled by each participant during the following session.

\section{Data collection}

\section{Demographic and records' data}

Demographic data included questions regarding age, gender, education, occupation, rural/urban place of living and smoking status. The data were collected from the patients' records. They included the duration of dialysis in months, patients' diabetes mellitus (DM) status and body mass index (BMI) and mean values of the following variables: HD adequacy index $(K t / V)$, C-reactive protein (CRP), viral hepatitis $\mathrm{C}$ and/or B status, hemoglobin, leukocyte, parathyroid hormone, calcium, alkaline phosphatase (ALP) and phosphorus levels. The mean values of the abovementioned variables for all patients were calculated 3 months preceding the examination. 


\section{Oral examination and scales: OHIP-I4 and GOHAl}

The oral examination was carried out in natural daylight with a plane mirror (E. Hahnenkratt GmbH, Königsbach-Stein, Germany) and a $0.5 \mathrm{~mm}$ ball-ended periodontal probe (LMInstruments Oy, Parainen, Finland) according to the WHO criteria at the bedside during an HD session. ${ }^{24}$ The number of remaining teeth, teeth with dental caries (D), missing teeth (M) and fillings (F) was recorded, and the decayed, missing and filled teeth (DMF-T) Index was determined. The plaque and the calculus were estimated only for participants with dentition (59) using the Simplified Oral Hygiene Index (OHI-S) and the Plaque Index (PI) ${ }^{25,26}$ The periodontal health was assessed using the Gingival Index (GI). ${ }^{27}$

For the evaluation of OHRQoL, the Polish version of the OHIP-14 and GOHAI scales was used. They were validated in an earlier survey. ${ }^{28}$ The responses in the OHIP-14 and GOHAI scales were based on the 5-point Likert scale (0 - never, 4 - always), and patients could select one out of five answers. The OHIP-14 scale ranged from 0 to 56 , and the GOHAI scale ranged from 0 to 48. Higher scores indicated poorer OHRQoL. Apart from the questionnaires assessing the OHRQoL, patients were asked to answer questions regarding their status of oral health (bad/fair/ good), the occurrence of dry mouth and chewing ability (yes/no), the presence or absence of partial/complete dentures, the needs of dental treatment (yes/no) and the smoking status.

\section{Data analysis}

The OHIP-14 and GOHAI scales were obtained by adding up the response codes of 14 items for OHIP-14 and 12 items for GOHAI constituting the measure. Descriptive statistics were presented as mean, median and quartile values. The Kruskal-Wallis test and the Mann-Whitney $U$ test were used to compare the OHIP-14 and GOHAI scores in relation to self-ratings of oral health, education, chewing ability and dry mouth. The OHIP-14 and GOHAI scores were dichotomized using median splits. Relationships between categorical variables were assessed using Pearson's $\chi^{2}$ tests. Spearman's rank correlation coefficients were used to measure the relationships between OHIP-14 and GOHAI scores and OHI-S PI and GI. The values of Cronbach's alpha were calculated to assess the internal consistency. The statistical analysis was performed using the IBM SPSS Statistics 20.0 software (IBM Corporation, Armonk, NY, USA). Statistical hypotheses were verified with a significance level of $<0.05$.

\section{Results}

The mean duration of HD treatment was 43.8 months and ranged between 1 and 264 months. Even though $81.9 \%$ of the participants were dentate, only $22.2 \%$ of the respondents had $>20$ teeth. The mean number of teeth was 10.9 . Among the dentate subjects, $44.1 \%$ wore removable dental prostheses, with more women $(60.7 \%)$ than men $(29.0 \%)$. The relationships between the GOHAI and OHIP-14 scales and the biochemical and clinical parameters, namely, CRP, hemoglobin, leukocyte, parathyroid hormone, calcium, ALP, phosphorus levels, $K t / \mathrm{V}$, hepatitis $\mathrm{C}$ virus (HCV), hepatitis $\mathrm{B}$ virus (HBV) status, DMF-T, D, M, F and BMI did not reach the statistical significance. A significant difference was observed for BMI $(p<0.001)$ between HD patients with and without DM.

Table 1 lists the percentage of patients who responded always, often and sometimes to each GOHAI question. The most prevalent dimensions in the GOHAI scale were psychological impacts (94.4\%) represented by the items unhappy with appearance, worried or concerned, nervous or selfconscious and uncomfortable eating in front of people and functional limitations (88.9\%) represented by trouble biting/ chewing food, uncomfortable to swallow and prevented from speaking. Looking at items separately, the most prevalent were uncomfortable to swallow and discomfort when eating and unhappy with appearance. In our study, none of the subjects scored the maximum in either measure. The GOHAI score ranged between 13 and 41, and did not reveal subjects without any oral health problems.

Table I Percentage of subjects responding always, often and sometimes to each GOHAl item

\begin{tabular}{|c|c|}
\hline GOHAI & $\%$ \\
\hline Functional limitation $^{a}$ & 88.9 \\
\hline Trouble biting/chewing food & 44.4 \\
\hline Uncomfortable to swallow & 70.8 \\
\hline Prevented from speaking & 25.0 \\
\hline Pain and discomfort ${ }^{a}$ & 84.7 \\
\hline Discomfort when eating & 63.9 \\
\hline Use medication to relieve pain & II.I \\
\hline Teeth, gums, sensitive to hot/cold & 33.3 \\
\hline Psychological impacts $^{a}$ & 94.4 \\
\hline Unhappy with appearance & 66.7 \\
\hline Worried or concerned & 36.1 \\
\hline Nervous or self-conscious & 25.0 \\
\hline Uncomfortable eating in front of people & 20.8 \\
\hline Behavioral impacts $^{a}$ & 30.6 \\
\hline Limit kinds or amounts of food & 22.2 \\
\hline Limit contact with others & 6.9 \\
\hline
\end{tabular}

Note: ${ }^{\text {aThe }}$ percentage of problems within a dimension was calculated as a percentage of patients having problems with at least one item in a given dimension. Abbreviation: GOHAl, Geriatric/General Oral Health Assessment Index. 
Table 2 lists the percentage of patients who responded always, often and sometimes to each OHIP-14 question. The most prevalent dimensions in the OHIP-14 scale were psychological impacts $(62.5 \%)$ represented by the items been self-conscious, difficult to relax, been embarrassed, felt life less satisfying and pain and discomfort (52.8\%) represented by painful aching in mouth and uncomfortable to eat foods. Looking at items separately, the most prevalent were uncomfortable to eat foods, and diet has been unsatisfactory. The OHIP-14 score ranged from 0 to 47 . In the OHIP-14 scale, there were 17 participants who did not report any oral health problems.

The GOHAI (mean 14.71; SD 7.21) and the OHIP-14 (mean 8.87; SD 10.95) scores were reflected in their median values of 13 (lower quartile 9; upper quartile 19) and 4 (lower quartile 1; upper quartile 13), respectively. The internal reliability (Cronbach's alpha) was 0.637 for GOHAI and 0.918 for OHIP-14.

Table 3 lists the mean, median and quartile values of the GOHAI and the OHIP-14, and particular grouping variables. Demographic data such as gender, education, occupation and rural/urban place of living were not related to either the GOHAI or the OHIP-14. The duration of HD was not significantly correlated with the GOHAI and the OHIP-14. The $\mathrm{D}, \mathrm{M}, \mathrm{F}$ and DMF-T index did not correlate with any of the scales. Only the chewing problems declared by participants were significantly related to the higher scores of GOHAI

Table 2 Percentage of subjects responding always, often and sometimes to each OHIP-I4 item

\begin{tabular}{|c|c|}
\hline OHIP-I4 & $\%$ \\
\hline Functional limitation $^{a}$ & 44.4 \\
\hline Trouble pronouncing words & 20.8 \\
\hline Sense of taste worse & 19.4 \\
\hline Pain and discomfort ${ }^{a}$ & 52.8 \\
\hline Painful aching in mouth & II.I \\
\hline Uncomfortable to eat foods & 30.6 \\
\hline Psychological impacts ${ }^{a}$ & 62.5 \\
\hline Been self-conscious & 27.8 \\
\hline Felt tense & 27.8 \\
\hline Difficult to relax & 13.9 \\
\hline Been embarrassed & 20.8 \\
\hline Felt life is less satisfying & 22.2 \\
\hline Behavioral impacts $^{a}$ & 45.8 \\
\hline Diet has been unsatisfactory & 30.6 \\
\hline Had to interrupt meals & 16.7 \\
\hline Been irritable with others & 12.5 \\
\hline Difficulty doing usual jobs & 6.9 \\
\hline Totally unable to function & 6.9 \\
\hline
\end{tabular}

Note: ${ }^{\text {aT }}$ The percentage of problems within a dimension was calculated as a percentage of patients having problems with at least one item in a given dimension. Abbreviation: OHIP-I4, Oral Health Impact Profile.
( $p=0.001)$ and OHIP-14 $(p<0.001)$ scales. Moreover, the higher OHIP-14 scores were significantly associated with the dental treatment needs $(p=0.029)$ and poor self-rated oral status $(p=0.001)$.

\section{Discussion}

The evaluation of OHRQoL not only focused on traditional medical/dental assessment but also shifted toward social, emotional and physical functioning of a person. ${ }^{18}$ Our study is one of the first conducted in Europe that estimates which scale describing OHRQoL is more useful in maintenance HD patients. This study shows that OHRQoL enhances our understanding regarding the relationship between patients' oral health and general health, meaning the end-stage renal disease in this particular group.

In our study, we did not find any close correlation between the results of clinical evaluation carried out by the dentist and the patient's subjective assessment. It is to be emphasized that the clinical evaluation showed an unsatisfactory oral status of the examined people (the presence of teeth with caries, dental deposits and gingivitis). Therefore, a dental examination should be an inherent element in the assessment of OHRQoL in HD patients.

We found that psychological aspects were the important factors among all dimensions in the GOHAI and OHIP-14 scales in HD patients. According to the literature, the OHIP-14 gives a greater weight to psychological and behavioral outcomes, while the GOHAI gives a greater weight to functional limitation, pain and discomfort. ${ }^{18,20,21}$ However, the most common problems revealed by the GOHAI scale were those concerning uncomfortable to swallow and unhappy with appearance of teeth and gums. Moreover, in the OHIP-14 scale, the most common problems concerned discomfort while eating and non-satisfactory diet. In our opinion, these findings reflect the diminished number of teeth in the examined group. At the mean age of 63 years, the HD patients had on average 10 teeth and almost 2 teeth with cavities. Unquestionably, the masticatory efficiency was not maintained. On the other hand, it was surprising that the OHIP-14 scale captured $23.6 \%$ of participants who did not report any oral health problems in this situation. This was not reflected in the GOHAI scale. According to Gerritsen et al, ${ }^{23}$ the number of missing teeth affects the OHRQoL. Our research confirmed the findings of Schmalz et $\mathrm{al}^{9}$ that dental and periodontal findings did not significantly affect the OHRQoL. Moreover, in our survey, it was proven in both scales. One explanation is that the HD patients might have accepted their impaired level of oral health over the years. Another explanation, 
Table 3 Mean, median and quartile values of the GOHAI and OHIP-I4 scores of grouping variables

\begin{tabular}{|c|c|c|c|c|}
\hline Variables/n & $\begin{array}{l}\text { GOHAI - mean; } \\
\text { median (QI-Q3) }\end{array}$ & $p$-value & $\begin{array}{l}\text { OHIP-I4 - mean; } \\
\text { median (QI-Q3) }\end{array}$ & $p$-value \\
\hline \multicolumn{5}{|l|}{ Smoking status } \\
\hline $\mathrm{No} / 54$ & |4.3।; | $3.00(9.00-19.00)$ & 0.519 & $8.85 ; 4.00(0.00-12.25)$ & 0.420 \\
\hline Yes/18 & I5.89; | $4.50(9.00-22.25)$ & & $8.94 ; 6.50(1.00-15.75)$ & \\
\hline \multicolumn{5}{|l|}{ Dental status } \\
\hline Dentate/59 & $15.24 ; 14.00(10.00-20.00)$ & 0.113 & $9.61 ; 5.00(1.00-17.00)$ & 0.132 \\
\hline Edentate/I3 & I2.3I; $9.00(8.00-16.50)$ & & $5.54 ; 2.00(0.00-9.50)$ & \\
\hline \multicolumn{5}{|c|}{ Number of teeth } \\
\hline$<20 / 55$ & I4.84; I3.00 (9.00-21.00) & 0.755 & $10.33 ; 7.00(1.00-17.00)$ & 0.058 \\
\hline$\geq 20 / 17$ & I4.29; $14.00(12.00-17.50)$ & & $4.18 ; 2.00(0.50-4.50)$ & \\
\hline \multicolumn{5}{|c|}{ Dental treatment needs } \\
\hline $\mathrm{No} / 35$ & $12.91 ; 12.00(8.00-16.00)$ & 0.071 & $6.31 ; 2.00(0.00-9.00)$ & 0.029 \\
\hline Yes/37 & $16.41 ; 14.00(10.00-23.50)$ & & II.30; $6.00(2.00-19.00)$ & \\
\hline \multicolumn{5}{|l|}{ Chewing ability } \\
\hline Yes/22 & 19.86; $22.00(11.50-28.00)$ & 0.001 & $19.50 ; 20.00(7.00-27.50)$ & 0.000 \\
\hline $\mathrm{No} / 50$ & $12.44 ; 12.50(8.75-15.25)$ & & $4.20 ; 2.00(0.00-6.25)$ & \\
\hline \multicolumn{5}{|l|}{ Dry mouth } \\
\hline Yes/42 & I5.10; $13.00(9.00-21.00)$ & 0.731 & $9.50 ; 5.00(0.75-17.25)$ & 0.461 \\
\hline $\mathrm{No} / 30$ & I4.17; I3.50 (9.00-16.50) & & $8.00 ; 4.00(0.75-17.25)$ & \\
\hline \multicolumn{5}{|c|}{ Self-rated oral status } \\
\hline Poor/13 & $19.00 ; 19.00(10.50-27.00)$ & 0.063 & $16.38 ; 18.00(6.00-25.50)$ & 0.001 \\
\hline Fair/3I & $14.84 ; 14.00(10.00-19.00)$ & & $8.65 ; 4.00(1.00-12.00)$ & \\
\hline Good/28 & $12.57 ; 12.00(8.00-16.00)$ & & $5.64 ; 1.50(0.00-7.00)$ & \\
\hline \multicolumn{5}{|l|}{$\mathrm{PI}$} \\
\hline $0-1.0 / 14$ & 14.84; 15.00 (II.50-19.25) & 0.987 & $12.00 ; 3.00(0.75-20.75)$ & 0.685 \\
\hline $1.1-2.0 / 20$ & $15.35 ; 14.00(9.50-20.50)$ & & $7.95 ; 4.00$ (2.00-11.25) & \\
\hline $2.1-3.0 / 25$ & I5.44; I 3.00 (9.50-23.50) & & $10.88 ; 7.00(1.50-17.50)$ & \\
\hline \multicolumn{5}{|c|}{ 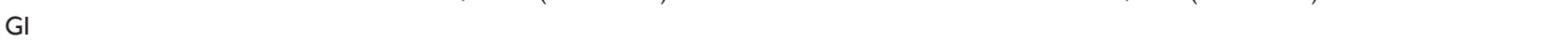 } \\
\hline $0-1.0 / 27$ & $14.84 ; 14.00(10.00-18.00)$ & 0.866 & $8.65 ; 3.00(1.00-9.00)$ & 0.200 \\
\hline $1.1-2.0 / 26$ & I5.89; $14.00(10.50-24.50)$ & & $9.61 ; 6.50(0.75-20.00)$ & \\
\hline $2.1-3.0 / 6$ & $16.38 ; 14.00(5.00-26.75)$ & & $10.33 ; 7.00(3.50-30.50)$ & \\
\hline \multicolumn{5}{|l|}{ OHI-S } \\
\hline $0-1.2 / 17$ & 13.06; $14.00(11.50-16.00)$ & 0.171 & $4.29 ; 1.00(0.00-4.50)$ & 0.09 \\
\hline $1.3-3.0 / 24$ & $17.33 ; 15.00(12.25-23.25)$ & & $12.00 ; 7.50(3.25-18.00)$ & \\
\hline $3.1-6.0 / 18$ & $14.50 ; 12.00(8.00-26.00)$ & & $11.44 ; 7.00(1.75-20.75)$ & \\
\hline
\end{tabular}

Abbreviations: GOHAI, Geriatric/General Oral Health Assessment Index; OHIP-I4, Oral Health Impact Profile; PI, Plaque Index; Gl, Gingival Index; OHI-S, Simplified Oral Hygiene Index.

more general, suggests that as a result of limited number of teeth, there was a slow, gradually progressive change in food choice. ${ }^{29}$ Moreover, when a serious general disorder affecting the whole body appears, the oral health problems are no longer a major concern..$^{9,12}$ A similar observation that the OHIP-14 scale had more impact on OHRQoL was made by Guzeldemir et al. ${ }^{5}$ Compared to our findings, they did not provide any significant correlations between the scales and the clinical variables. On the other hand, the mean values of the GOHAI and the OHIP-14 obtained in community dwelling subjects from the same region were higher than in HD patients. ${ }^{28}$ Such a large span of OHIP-14 values in various studies may show a high variability in the approach to oral health in HD patients. Furthermore, it may indicate that the subjective scales are differently perceived by healthy people and chronically ill patients, and that other factors, not directly related to oral health (eg, psychological factors), may affect the final results.

HD patients declared chewing problems, which were reflected in both scales. This confirms that the chewing ability is perceived as an important determinant of oral health. ${ }^{30}$ Chewing problems in HD patients should arouse concern. The difficulty in eating solid foods may lead to malnutrition and underweight, and consequently have an impact on the survival. Chewing affects the quality and quantity of food intake, and may play an underestimated role in strengthening malnutrition, which is a component of the malnutrition, inflammation and atherosclerosis (MIA) syndrome in HD patients. ${ }^{31,32}$ Chewing problems are quite often related to dry mouth/xerostomia. This symptom is frequently occurring in 
HD patients. The saliva secretion in these patients is lower than in the general population. ${ }^{33}$ Moreover, these patients are required to limit their water intake. ${ }^{32}$ However, the feeling of dry mouth in our participants did not correlate with any of the scales. HD patients probably adapted to dry mouth and did not perceive dryness as a negative syndrome.

The clinical assessment is an essential part of subjective oral health measures. Moreover, we strongly support the indications presented by Bayraktar et $\mathrm{al}^{8}$ that there should be cooperation between nephrologists and dentists to consult and treat the systemic conditions of patients receiving an HD therapy. Worth emphasizing is also the role of periodontal treatment in reducing the moderate systemic inflammatory response, which plays a key role in the progression of cardiovascular system diseases, being one of the main causes of deaths in the population of dialyzed patients. ${ }^{34-36}$

\section{Strengths and limitations}

As far as we know, this is the first study that evaluates which OHRQoL scale is more useful in maintenance HD patients. We admit that the small sample size is a limitation of this study, but our participants represented the population of chronically HD patients in Bialystok - a city with $<300,000$ inhabitants. An inevitable strength of our study is a very high response rate up to $78.2 \%$. Another strength is that we included edentulous patients and patients with few teeth who are sometimes excluded from the surveys. ${ }^{9,10}$ It was proved that missing teeth negatively influenced the OHRQoL. ${ }^{23}$ The lack of generalization of the results may be considered as a limitation of our study. However, we mainly concentrated on the comparison of two well-established measures in this specific group of patients. In our opinion, a further generalization of the results requires a confirmation on a larger sample.

\section{Conclusion}

The HD patients had an unsatisfactory oral status, but using only OHRQoL scales was insufficient to capture all their oral health problems. The scales did not fully reflect poor oral health in HD patients. The oral problems were not a major concern for this group of patients, which could indicate the adaptation to impaired oral health or a change of health priorities. Regular dental examinations together with the assessment of OHRQoL in HD patients are required for a comprehensive patients' state. In our study, more variables were significantly related to the OHIP-14 scale than to the GOHAI scale. Thus, the OHIP-14 scale may be more useful in assessing OHRQoL in HD patients.

\section{Author contributions}

The manuscript has been read and approved by all authors. The authors are alone responsible for the content and writing of the paper. All authors contributed toward data analysis, drafting and revising the paper and agree to be accountable for all aspects of the work.

\section{Disclosure}

The authors report no conflicts of interest in this work.

\section{References}

1. Preamble to the Constitution of the World Health Organization as Adopted by the International Health Conference, New York, June 19-22, 1946.

2. Craig RG. Interactions between chronic renal disease and periodontal disease. Oral Dis. 2008;14(1):1-7.

3. Akar H, Akar GC, Carrero JJ, Stenvinkel P, Lindholm B. Systemic consequences of poor oral health in chronic kidney disease patients. Clin J Am Soc Nephrol. 2011;6(1):218-226.

4. Chuang SF, Sung JM, Kuo SC, Huang JJ, Lee SY. Oral and dental manifestations in diabetic and nondiabetic uremic patients receiving hemodialysis. Oral Surg Oral Med Oral Pathol Oral Radiol Endod. 2005;99(6):689-695.

5. Guzeldemir E, Toygar HU, Tasdelen B, Torun D. Oral health-related quality of life and periodontal health status in patients undergoing hemodialysis. J Am Dent Assoc. 2009;140(10):1283-1293.

6. Pakpour AH, Kumar S, Fridlund B, Zimmer S. A case-control study on oral health-related quality of life in kidney disease patients undergoing haemodialysis. Clin Oral Investig. 2015;19(6):1235-1243.

7. Bayraktar G, Kurtulus I, Kazancioglu R, et al. Evaluation of periodontal parameters in patients undergoing peritoneal dialysis or hemodialysis. Oral Dis. 2008;14(2):185-189.

8. Bayraktar G, Kurtulus I, Duraduryan A, et al. Dental and periodontal findings in hemodialysis patients. Oral Dis. 2007;13(4):393-397.

9. Schmalz G, Kollmar O, Vasko R, Müller GA, Haak R, Ziebolz D. Oral health-related quality of life in patients on chronic haemodialysis and after kidney transplantation. Oral Dis. 2016;22(7):665-672.

10. Borawski J, Wilczyńska-Borawska M, Stokowska W, Myśliwiec M. The periodontal status of pre-dialysis chronic kidney disease and maintenance dialysis patients. Nephrol Dial Transplant. 2007;22(2): 457-464.

11. Gavaldá C, Bagán J, Scully C, Silvestre F, Milián M, Jiménez Y. Renal hemodialysis patients: oral, salivary, dental and periodontal findings in 105 adult cases. Oral Dis. 1999;5(4):299-302.

12. Klassen JT, Krasko BM. The dental health status of dialysis patients. $J$ Can Dent Assoc. 2002;68(1):34-38.

13. Teratani G, Awano S, Soh I, et al. Oral health in patients on haemodialysis for diabetic nephropathy and chronic glomerulonephritis. Clin Oral Investig. 2013;17(2):483-489.

14. Bots CP, Poorterman JH, Brand HS, et al. The oral health status of dentate patients with chronic renal failure undergoing dialysis therapy. Oral Dis. 2006;12(2):176-180.

15. Bayraktar G, Kazancioglu R, Bozfakioglu S, Yildiz A, Ark E. Evaluation of salivary parameters and dental status in adult hemodialysis patients. Clin Nephrol. 2004;62(5):380-383.

16. Cavuoti S, Matarese G, Isola G, Abdolreza J, Femiano F, Perillo L. Combined orthodontic-surgical management of a transmigrated mandibular canine: a case report. Angle Orthod. 2016;86(4):681-691.

17. Cutroneo G, Piancino MG, Ramieri G, et al. Expression of musclespecific integrins in masseter muscle fibers during malocclusion disease. Int J Mol Med. 2012;30(2):235-242.

18. Sischo L, Broder HL. Oral health-related quality of life: what, why, how, and future implications. J Dent Res. 2011;90(11):1264-1270. 
19. Slade GD. Derivation and validation of a short-form oral health impact profile. Community Dent Oral Epidemiol. 1997;25(4):284-290.

20. Atchison KA, Dolan TA. Development of the geriatric oral health assessment index. J Dent Educ. 1990;54(11):680-687.

21. Slade GD, Spencer AJ. Development and evaluation of the oral health impact profile. Community Dent Health. 1994;11(1):3-11.

22. Locker D, Matear D, Stephens M, Lawrence H, Payne B. Comparison of the GOHAI and OHIP-14 as measures of the oral health-related quality of life of the elderly. Community Dent Oral Epidemiol. 2001;29(5): 373-381.

23. Gerritsen AE, Allen PF, Witter DJ, Bronkhorst EM, Creugers NH. Tooth loss and oral health-related quality of life: a systematic review and meta-analysis. Health Qual Life Outcomes. 2010;5(8):126.

24. WHO. Oral Health Surveys: Basic Methods. 5th ed. Geneva: WHO; 2013. ISBN: 9789241548649.

25. Greene JC, Vermillion JR. The simplified oral hygiene index. $J A m$ Dent Assoc. 1964;68:7-13.

26. Silness J, Loe H. Periodontal disease in pregnancy. II. Correlation between oral hygiene and periodontal condition. Acta Odontol Scand. 1964;22:121-135.

27. Löe H, Silness J. Periodontal disease in pregnancy. Acta Odontol Scand. 1963;21:533-551.

28. Rodakowska E, Mierzyńska K, Bagińska J, Jamiołkowski J. Quality of life measured by OHIP-14 and GOHAI in elderly people from Bialystok, north-east Poland. BMC Oral Health. 2014;20(14):106.
29. Millwood J, Heath MR. Food choice by older people: the use of semistructured interviews with open and closed questions. Gerodontology. 2000; $17(1): 25-32$

30. Listl S. Chewing abilities of elderly populations in Europe. Int Dent $J$. 2011;61(4):175-178.

31. Tonbul HZ, Demir M, Altintepe L, et al. Malnutrition-inflammationatherosclerosis (MIA) syndrome components in hemodialysis and peritoneal dialysis patients. Ren Fail. 2006;28(4):287-294.

32. Chen LP, Chiang CK, Chan CP, Hung KY, Huang CS. Does periodontitis reflect inflammation and malnutrition status in hemodialysis patients? Am J Kidney Dis. 2006;47(5):815-822.

33. Kao CH, Hsieh JF, Tsai SC, Ho YJ, Chang HR. Decreased salivary function in patients with end-stage renal disease requiring hemodialysis Am J Kidney Dis. 2000;36(6):1110-1114.

34. United States Renal Data System. Excerpts from USRDS 2009 Annual Data Report. U.S. Department of Health and Human Services. The National Institutes of Health, National Institute of Diabetes and Digestive and Kidney Diseases. Am J Kidney Dis. 2010;55(Suppl 1): S1-S420.

35. D'Aiuto F, Ready D, Tonetti MS. Periodontal disease and C-reactive protein-associated cardiovascular risk. J Periodontal Res. 2004;39(4): 236-241.

36. Almeida S, Figueredo CM, Lemos C, Bregman R, Fischer RG. Periodontal treatment in patients with chronic kidney disease. A pilot study. J Periodontal Res. 2017;52(2):262-267.
Patient Preference and Adherence

\section{Publish your work in this journal}

Patient Preference and Adherence is an international, peer-reviewed, open access journal that focuses on the growing importance of patient preference and adherence throughout the therapeutic continuum. Patient satisfaction, acceptability, quality of life, compliance, persistence and their role in developing new therapeutic modalities and compounds to optimize

\section{Dovepress}

clinical outcomes for existing disease states are major areas of interest for the journal. This journal has been accepted for indexing on PubMed Central. The manuscript management system is completely online and includes a very quick and fair peer-review system, which is all easy to use. Visit http://www. dovepress.com/testimonials.php to read real quotes from published authors. 